\title{
REVIEW OF GEOPHYSICAL STRAIN MEASUREMENT
}

\author{
P. H. Sydenham*
}

\section{Synopsis}

Strain measurements are of interest to both fundamental and engineering geophysics. A review of the various methods for surface and sub-surface deformation measurement is presented. The need to improve testing and calibration methods is stressed.

\section{Introduction}

Strain measurements in geophysics are becoming increasingly important. Measurements are predominantly of linear strain although volume strain measurement has been attempted in a few instances.

In earthquake studies it is hoped that a knowledge of strain rate will enable earthquakes to be predicted by monitoring strain build-up in the earth. Reid's elastic rebound theory, generally accepted as a possible mechanism in earthquakes, implies that stress increase is relieved by inelastic earth movement at faults. Although stress may be the ideal parameter to instrument for, strain is at present the most practical to measure(1).

One aim of earthquake engineering is to produce structures that can withstand the expected magnitude shocks. To this end strain measurements on foundations and superstructures can assist further designs. Closely allied is the investigation of the degree of uniformity of rock strains induced by earthquake transients and by static loads imposed by large civil engineering projects, such as dams, and in mining operations whose gallery support-pillar stresses must be closely monitored.

In fundamental studies of earth tidal phenomena, strain determinations supplement those of gravitational changes and inclination of the vertical. Strain measurements are very relevant to the study of the earth free-modal vibrations (1).

Relative length changes over kilometre distances enable determination of drift between continental masses, rift valley movements and tectonic movements in seismically active areas. Experiments involving land mass separation have been proposed to study the widening of the Red Sea $(2)$ and movements in New Guinea 3 ). Glacial moment is also monitored by relative position changes of markers placed in the ice. These examples are all dynamic length changes having extremely slow velocities.

* Lecturer in Geophysical Instrumentation, Department of Geophysics, University of New England, Armidale, N.S.W., 2351, Australia.
Across the globe geodetic control nets have been established within which continental locations are determined. At present these nets have precisions around one part in $10^{6}$, with subsequent improvement in the accuracy of surface measurements of those nets it will be necessary to gain a quantitative knowledge of the change of global shape with time. Already some geodesists are correcting their measurements for tidal deformation in levelling operations.

An allied scientific technique that may be overlooked is Very Long Baseline Interferometry (VLBI for short). This method was originated for investigating the size of stellar sources. It has, however, the potential to measure length changes of baselines spanning continents to one part in $10^{9}$.

In industrial engineering, creep measurements of engineering materials involves continuous length change measurements performed over periods as long as 100,000 hours, 111.4 years) to precisions of a little better than one part in $10^{\circ}$.

In each of the above, the strain measurement methods required are characterized by the stringent requirements of extreme stabilities over times ranging from days to years with resolutions ranging from one part in $10^{6}$ in materials testing and surface earth measurements down to one part in $10^{11}$ in underground tidal extensometers. Velocities are slow. Tidal strain. for instance, changes at a rate of $1 \mathrm{~m}$ in $10^{4}$ years.

Requirements for good geophysical strain meters are in many respects those of inter. national length standards. At present National decametre standards are barely sufficient in precision for strain interval absolute length measurement. If absolute length could be measured to a part in $10^{8}$ over long distances with equal global precision it would be possible to reduce the number of continuously monitoring strain meters needed for global coverage: bench marks could be remeasured periodically. Recent work with absorption-cell stabilized lasers indicates that this will soon be feasible. In the mean-time we are left with the Krypton interferometer standard which must be cascaded from centimetres to decametres by a laborious manual process that loses much of the inherent accuracy of the fundamental standard.

It can be seen from the brief discussion of geophysical strain that it covers a widespread interest from many different aspects of science and engineering.

This paper is concerned with measurement 
techniques for determining high-resolution relative length changes over extreme periods of time. By presenting different view points and approaches it is hoped those associated with strain measurement will see the relevance of previously unused methods in their own area of interest. Long-length standards are briefly discussed as these are most important to u1tralong period strain determination. Finally, a description of a current programme of the University of New England, Australia, is outlined to show its relation to the overall problem of strain-meter precision.

\section{General Comments on Strain Measuring Instruments}

Measurement methods divide broadly into those which can resolve earth tidal amplitudes with ease, and usually have enough sensitivity to record earthquake strain oscillations, and those which cannot. The diurnal solid-tide strains amplitude is between 1 to 5 parts in $10^{8}$ depending upon geographical location and orientation. Strains due to earthquakes range from this to one to two orders lower. Figure 1 shows a tidal wave with a magnitude 6 earthquake. With few exceptions underground instal1ation is needed to enable these sensitivities to be used as surface effects produce strains which would completely mask the tidal waves and earth oscillations。

The second group of instruments are those designed for surface measurements as are found in geodetic surveying. These instruments cannot resglve tides at this time, having one part in $10^{7}$ resolution at the best.

Linear strain instruments consist of an adequately stable standard of length which is mounted or utilized so that its length can be compared against the unknown changing length variable. Comparison can be by manual means or more preferably by some combination of electro/optical/mechanical transducers from which a continuous record can be made。

The standard of length is either a rigid or flexible mechanical member or, alternatively, it may be some feature of the transmission or of the coherent properties of electromagnetic (EM) radiation at optica1, near optical or microwave frequencies.

Although the time stability of the chosen standard in a strain measuring method is of the utmost importance, little emphasis seems to have been placed on establishing independant means to assess the stability of the mechanical tidal instruments which have been installed. Nor have attempts been made to calibrate the standards absolute length which would serve this purpose. Absolute length knowledge of the standard is important in the investigation of the secular drift of the measured variable and vital if the measuring method is discontinuous with time.

In mechanical-standard methods the relative length changes are now most easily measured with inductive or capacitive direct gauging systems, each being capable of $10^{-9} \mathrm{~m}$ resolution or better. Inductive gauges are less affected by humidity and considerably simpler to set up. There is now no real need for mechanical amplification of strain meter movements. As electric transducers are extremely robust the mechanical strain meter can now be made to be extremely reliable and simple to construct. thigh resolution tm techniques the basic radiation wavelength, or a superimposed modulation wavelength, is used as a standard for determining distance changes. In these methods it is firstly necessary to monitor the complete cyclic changes occurring and then to interpolate the remaining fringe interval into as many as 100 to 1000 parts。 In tidal-sensitivity strain-meters (the laser interferometer) the interpolation presents a major part of the complete system design.

Absolute measurement of decametric intervals on the earth or of the standard itself to better than a part in $10^{6}$ is a formidable problem at this time but increasing interest in these longer ranges has prompted increased research by National bureaux.

Mechanical methods are now discussed in detail, following on with those using radiation.

\section{Mechanical Methods}

The first strain meter operating with tidal sensitivities was a mechanical system devised by Benioff around $1930(4)$. It was built in Pasadena, U.S.A. His instrument, illustrated in figure 2 , consisted of $2 \mathrm{~m}$ lengths of 12 in. diameter pipe set vertically into a granite bed with concrete. These pillars were separated by a nominal distance of $20 \mathrm{~m}$. Supported horizontally between these pillars by wire slings was a dength of 2 in. diameter steel pipe insulated to gain a longer thermal time-constant. One end was fastened to a pillar. The gap variation at the other end was monitored by an electromagnetic transducer of Benioff's design.

A somewhat similar standard bar concept had been used by Milne in Japan around 1888 (4) but this had insufficient amplification. (A displacement magnification of $10^{6}$ is needed with this gauge length to produce an adequately large record.)

The performance of this gauge (often called a Benioff instrument) can be bettered by using a length standard having a lower thermal coefficient of expansion. For this reason steel (thermal expansion coefficient of 12 $\left.10^{-6} /{ }^{\circ} \mathrm{C}\right)$ has been replaced by fused silica or invar rods (coefficient of $0.210^{-6} /{ }^{\circ} \mathrm{C}$ )。 This reduces the temperature errors in the standard length but does not eliminate them entirely as variations in deep tunnels can exceed $1^{\circ} \mathrm{C}$ due to inducted heat via body water or, perhaps, geothermal waves。 Bepioff reported quartz instruments in $1959^{(5)}$ which are installed in Southern California, U.S.A.

Similar instruments using $200 \mathrm{ft}$. tubes have been construced at Ogdensburg, New Jersey, U.S.A. by the staff of Columbia University. These were reported in $1964(6)$.

A survey of global tidal stations has recently been made by Bilham where he has catalogued $(7)$ around 200 strain gauges of one form or another.

A reuseable Benioff gauge has been reported by Blayney and Gilman(8) in 1965. Their instrument is built in the Kipapa Tunnel in Hawaii. This is claimed to be semi-portable as it can be dismantled for resiting. It is 
interesting to conject if this instrument could be reassembled with equal stability and absolute length. This could be important if it were reused at a later date in a previously occupied location where records needed to be re-established.

Benioff instruments exceeding 200 ft. in length have been proposed along with thermally compensated equipment (9). As yet these do not seem to have been reported. Vertical models have been constructed.

An interesting mechanical amplification method is that of the rolling demorograph used by Japanese observers. This uses a quartz standard rod to roll a cylinder along a horizontal glass plate. An optical lever is then used to amplify the cylinder rotation. Russians have also used this technique(10). It is, however, only just capable of resolving earthtides but could find application in structure strain measurement for it is robust and can operate without electric power.

Solid mechanical standards have the advantage of relative cheapness and long-term reliability。 They are, however, plagued with lack of knowledge of secular length changes in the mechanical standard. It is surprising that so little study of this effect has been made with particular reference to strain meter applications. It seems that intercomparison between two similar instruments mounted side by side (transverse comparison) has not been reported. Direct thermal control of the standard is also a very practicable proposition. Temperature control to considerably less than $0.0010 \mathrm{C}$ seems realistic by inference from results of work on the temperature control of similar size objects $(11-13)$.

The disadvantage of the solid standard instrument is that the slender tube needs multiple supporting points. This restriction can be overcome by using a tensioned flexible mechanical member in wire form. These can be said to be truly portable instruments for the complete instrument (neglecting thermal insulation that may be needed) requires only $1 \mathrm{ft}^{3}$ space for transportation.

Invar wire, freely suspended in catenary has been used extensively for surface geodetic surveying from the $1880^{\circ} \mathrm{s}$ to the $1950^{\circ} \mathrm{s}$ but never with resolutions exceeding a part in $10^{6}$ due to calibration limitations. In 1951 Sassa and others published a method using a tensionedwire for measuring tidal strains (14). The Sassa extensometer, pictured in figure 3, consisted of a $25 \mathrm{~m}$ length of Super Invar (the lowest thermal expansion invar form) which was hung horizontally between rock-embedded supports. A $350 \mathrm{gm}$ weight was suspended by the centre and the wire adjusted to obtain a central sag of $1 / 70$ th of the span. The vertical movements between the weight and the bed-rock were recorded using biofilar mechanical amplification and an optical lever. This is similar to the sag-wire geodetic-base length-change measurement method $(15)$. In the latter, however, no central weight is added. This concept has inherent mechanical amplification for the central deflection is many times greater than the span change producing it. It does, however, require three terminal points which might introduce errors due to variation in elevation of each point due to base bend or mount creep.
A recently reported tensioned-wire mech. anical seismic strain-meter using a two point support is that due to Sydenham(16)。 In this method, see figure 4, a simple beam balance applies absolute axial tension to a freely suspended wire (invar and carbon fibres have been used to date). A Iinear variable differential transformer (1.v.d.t.) metrology probe was used to monitor the rotation of the beam being placed to gauge the end of the wire along its axis. (This reduces angular errors due to beam rotational inaccuracies.) The other end of the wire was fastened to the rock $10 \mathrm{~m}$ away. The original installation used expanding bolts for fastening at the terminals end was installed and recorded at tidal sensitivities within 6 hours of arrival at the site. These crude balances were originally built for investigating creep in long wires at controlled ambient temperatures(1?). They were subsequently used for earth tide measurement operating concurrently with the laser interferometer strain meter of the Cambridge Earth Strain Group. The two records produced in May 1969 have been published(18) showing that the inexpensive wire system ( $\$ 400$ ) gave performance similar to the laser. Extended processing of 7 months of records made with the balances operated by the Cambridge Group has shown that a drift rate of less than $10^{-7}$, per annum is being realised by the Invar wires(19). The carbon fibre, useful for its ability to produce a higher resonant frequency (17) system, has shown an order less stability (19).

The original units used ball pivot-bearings in the balance hinge. Gerard(20) has since built a number of superior balances using noncontact transduction and flexure pivots throughout. Damping is also incorporated. These are to be located in tunnels around, Wellington, New Zealand. Lurrent work at the University of New England, Australia, also uses strip hinges in their balances. Cambridge University have five units operating in their Queensbury tunnel, one system in a vertical bore hole, and one is ready for installation in an excavated trench in Iran (7, 19). Bilham has further investigated the application of wire strain meters in his thesis(7) where he describes an improved version which they have termed the Theometer. This uses flexure pivots, a non-contact 1.v.d.t. transducer, mechanical zero adjustment and operates close to optimally highest resonant frequency by using a flexible-beam support system which enables a large mass to act at a small radius, considerably reducing the rotational inertia (17). These improved balances are pictured diagrammatically in figure 5. The simplicity of the method and ease of the two point installation will enable rapid proliferation for more widespread studies of strain in both fundamental and engineering geophysics. An application involving three dimensional measurement and recording of bearing movements to .002 in. in $100 \mathrm{ft}$. lengths of a large electrical generating plant was proposed some time ago $(21)$.

With all mechanical standard methods more work is needed to establish the secular behaviour of the instrument. The least that should be done is to operate two instruments side by side.

If a sophisticated facility were 
realized by which the absolute length of the standard could be referred to the international length standards installations could be periodically reset to eliminate instrument drift thus gaining a clearer understanding of real-earth strains over prolonged periods and wide distributions. Absolute length is not vital but in the absence, at this time, of an adequately stable length of any sort it must be assumed the best long-term length that can be relied upon is the internationally agreed standard. As tensioned wires can be coiled for transport it is envisaged that those units might conceivably be calibrated to better than a part in $10^{?}$ at some central facility. This remains to be seen. Current designs of geodetic tapebases used by National Standards bodies would need to be modified or replaced entirely to give this a calibration as they are limited to just better than a part in $10^{6}$ by temperature variations, transfer mechanism errors and precision of the $4 \mathrm{~m}$ bar used to calibrate them.

One posible cause of secular length change in a mechanical standard is that brought about by changing stresses that alters the materials mechanical hysteretic condition. Once installed in strain meters, mechanical standards suffer virtually zero stress changes due to mechanical reasons. They are, however, still subject to temperature induced stress changes. It is interesting to conjecture on the stability of a mechanical standard that is controlled to an extremely fine temperature differential. A $12 \mathrm{~m}$ measuring base has been reported(11) that was temperature-controlled to $+0.03^{\circ} \mathrm{C}$ over periods of weeks. It was situated in a large surface civil engineering laboratory in which temperature differentials of $\pm 3^{\circ} \mathrm{C}$ were encountered in periods of minutes. Staff at the National Bureau of Standards have reported $(12,13)$ a large temperature-controlled water bath used for microcalorimetry. In this chamber temperatures are controlled to $\pm 2010-6 \circ \mathrm{C}$ per day. It is, therefore, to be expected that a thermally controlled mechanical base of invar or quartz, which is installed deep underground (to gain a slow predictable temperature gradient), might realise stabilities as high as parts in 1012 over considerable periods if a.c. thermometry control techniques are used. On such a base, if suitably decoupled from the earth, strain. meters could be checked for secular drift and calibrated in length. As well as acting as a stable base for comparison with strain meters it could directly monitor earth strain for it is, in fact, a stable length standard.

\section{Electromagnetic Radiation Methods-Interfero-Meters}

Electromagnetic distance measuring methods use one of three possibilities(22). (In each case the radiation is provided by the user whereas in a technique discussed below, use is made of naturally occurring radiation.)

a. Direct interferometry on the coherent properties of the radiation.

b. Phase comparison of a signal modulated on to the radiation.

c. Measurement of the transit time of the radiation.

The laser source provides long coherencelength radiation and has enabled very long
( $1 \mathrm{~km}$ has been built (23, 42)) strain meters to be realised. Laser strain meters using interferometric measurement have become very fashion. able and at least five $(7)$ are now in operation in mines and tunnels.

Basic laser cavities provide radiation having wavelength stability around a few parts in 10 ? at a cost of $\$ 1000$ 。 With lamb-dip stabilization and temperature control of the cavity the stability is bettered to a few parts in $10^{8}$ over periods of many hours $(24)$. The most recently devised stabilization method, and that certain to gain widespread adoption, uses an atomic absorption cell placed inside the laser cavity. The cavity length is automatically altered to keep the wavelength centred on an absorption line. The precision is equivalent to a part in 109 length error. One laser source using this method has recently been marketed for $\$ 7000$ 。

In laser strain meters the jaser is arranged to operate an interferometer on which fringe displacements are monitored as the long arm path-length alters. To reduce environmental effects of temperature, humidity and barometric pressure (which alters the air path refractive index and hence the wavelength) the long-arm, at least, needs to be in a roughly evacuated (10-5 atmos) or constant density tube。 Un-enclosed interferometers have been built where conditions allow. In normal atmospheres the laser method has a similar temperature co-efficient to quartz and is, therefore, similarly effected as are mechanical standard instruments.

With path lengths of $50 \mathrm{~m}$ the magnitude of an increment equal to $1 / 100$ th of tidal amplitude is around $1 / 100$ th of a fringe movement if a visible light source is used ( $\mathrm{He}-\mathrm{Ne}$ wavelength is approximately $0.633 \mu \mathrm{m})$. Secular drift and very-long period tidal components can be measured in terms of fringe counts but earthquake oscillations and daily tides need fringe interpolation to gain adequate resolution.

The first earth-strain interferometer appears to have been due to Vali and others $(29,30)$. They chose a Falry-Perot arrange. ment to enable the fringes to be interpolated most readily. They later intercompared the laser unit with a Benioff gauge (31) and with an inertial type of seismometer(32). The Michelson arrangement, figure 6, was used by the Cambridge earth-strain group (built in collaboration with the National Physical Laboratory $(18,33,34)$ ). This has fringe counting with digital interpolation to $\lambda / 8$ and anafogue resolution to $\lambda / 50(2.5$ parts in 1010$)$. Gerard provided an unmodulated output for the lamb-dip stabilized He-Ne laser $(35)$ and then added a displacement-balance fringe following advice(36) in which the far corner-cube reflector was servo-driven to keep the fringe at a reference position. Strain is measured as the output of the servo-loop. His fringefollowing method was complicated by the need to remove the modulation and the system subsequently was found to have too slow a response for following earthquake oscillations $(7)$. Absorption-cell stabilizing systems operate virtualiy at d.c. in which case the fringe following technique seems the better to use for interpolation purposes. Theoretical considerations show that due to noise fluctuations of the fringes there will be a definite chance of 
producing frequencies beyond the photodiode upper-frequency 1 imit. In this case a wrong incremental count might be registered. The N.P.L. laser has improved bandwidth and wide backlash zones to virtually eliminate this effect but for strain measurement the long periods involved increase the risk of fringe jumping. The University of Trieste also have a MicheIson interferometer in operation( 37 ).

Laser interferometers are by far the most expensive instrument $(\$ 6000-\$ 10,000)$ but they do have the advantage that their long-term absolute length stability appears to be superior to mechanical standards. This advantage, however, is currently much offset by the poor reliability of the laser source. Lives vary unpredictably from hours to thousands of hours. As a period of a year ( 8720 hrs) constitutes a continuous record of value for secular earthstrain measurement there is a high probability that the system will fail during a record run. With single frequency interferometric systems, the incremental nature implies that there is no way of re-establishing a continuous record without resort to hope or the use of a second unit overlapping (a cheap mechanical system?) the break in the record. Incorporation of a means to measure the whole arm length by traversing the far end reflector is a possible solution to this problem. Recent work with the gas stabilized lasers in strain meters $(38-40)$ should provide greater reliability as well as increased precision.

If the two arms of the interferometer are of approximately equal length, shorter coherence sources (for example, discharge lamp) can be used. Shamsi and stacey(41) have reported a shear strain meter using a mercury lamp in a quadrature spatial layout of a Michelson interferometer.

To date the longest interval strain meter is the $1 \mathrm{~km}$ instrument after Vali and Bostrom (23, 42). The longer the interval the less stringent are the stabilities of the terminal mounts of the equipment. With a $10 \mathrm{~m}$ interval a relative strain of $10^{-10}$ is $10^{-9} \mathrm{~m}$ which is still the dimension of atoms.) As Bender has already mentioned elsewhere in his review paper on lasers(2) for long-distance measurement, mechanical standard instruments could be made longer with relative ease if deemed necessary.

One method that has been proven capable of establishing the length of up to kilometre surface base lines to better than a part in 10 , is the light interference length multiplication method after V繁ilsal who proposed it as far back as $1923(43)$

The vâilsalla method is briefly as follows. (Figure 7 shows the principle). A one-metre quartz bar with polished and calibrated ends is standardized against the fundamental standard. The bar is placed in the apparatus within two mirrors. The next base point is prepared by measurement to get the following mirror in the approximately correct location. Radiation from a white 1 ight source is passed through the bar and also in a path adjacent to tne bar. The adjacent path is made an exact multiple of the internally multiply-reflected beam by interferometry. This establishes exact multiples of the rod-length which are extended linearly forward. The larger interval established is then used as the next to be multiplied.
In 1954 the International Association of Geodesy resolved in Rome that member countries should establish bases by this method for calibration of the faster and easier distance measuring methods $(44)$. To some extent this was adopted and the most recently established(45) is the Ohio base in the U.S.A. (1966)。

The extreme precision of the method makes it suited for absolute crustal deformation measurements of the highest order. It is, however, not an easy matter to establish fringes on long paths and it takes several. weeks to measure a baseline. Constructipnal details are given in the ohio base account(45).

Much of the literature on the Valis method is published over five languages. A few papers in English are available(46-49).

The alternative to this method of optical multiplication is to use fringes of superposition(22) to gain long lengths from short standards. This alternative seems similarly capable of a part in $10^{7}$ resolution but little use has been made of it.

\section{Electromagnetic Radiation Methods-Modulated Beam}

Bergstrand devised a modulated light beam method for measuring kilometre distances in 1949. The marketed devices are known as Geodimeters of which many models now exist. In these the radiation beam (from a discharge lamp or laser) is amplitude-modulated before being directed at a distant reflector - see figure 8. The received beam is compared in phase with the source of the modulation. The integral number of difference cycles and the relative phase of the remaining partial cycle provide a measure of range if the speed of light is known. Clark has summarized the principles and rield use of electronic surveying devices (50).

A technique using modulation of microwaves was discussed in South Africa by wadley in 1957. This, now known as the Tellurometer(50), can normally measure a greater range than the earlier optical instrument.

The most recently devised instrument using a modulated 1 ight beam is the Mekometer, after Froome and Bradse11 $(51,52)$. Here the beam is polarization rather than amplitude modulated. It is currently being placed on the market. The Mekometer covers distances to a kilometre or so with a correspondingly finer resolution $(0.1 \mathrm{~mm})$.

In modulation methods, as with direct interferometric means, the interval determined by the instrument depends greatly on the average path refractive index as this decides the mean EM velocity from which distance is inferred. In practice this limits the distance determination precision to a part or two in 106. Much research has been expended on determining ways to improve this precision for geodetic trilateral surveys and crustal strain measurement. For two different wavelengths of transmission the difference in transit time is a measure of the average path density. Systems using this foncept have been proposed and built $(53-57)$. Theoretical considerations laid down by McQuistan(58) show that the use of two different wavelengths should enable correction 
for path factors to a part in $10^{7}$. precision. Use of three wavelengths theoretically enables elimination of atmospheric errors. A dualfrequency system for fault zone monitoring
has been described by Owens and Earnshaw

Rift movements in Iceland were measured by Imperial College workers during 1969 onward with a Mark III Mekometer. Preliminary results, of this exercise $(59)$ indicate $1 \mathrm{~km}$ ines were measured to $0.3 \mathrm{~mm}$ probable error but there atmospheric conditions are very favourable.

Modulated methods are suited for surface deformation measurement。 In general they are not operated continuously but are used to measure lengths of an established network arranged over the area of interest. As they are expensive $\left(\$ 10,000^{\prime} \mathrm{s}\right)$ it is obviously impracticable to dynamically record all net.lengths simul taneously.

The latest Geodimeter incorporates a laser source to make it more versatile. To date, however, no dual frequency system has been marketed. The trade names Geodimeter and Tellurometer are used extensively as synonyms to describe optical and microwave instruments. There are, however, other marketed instruments which use similar techniques and have similar specifications.

The 1iterature on EM distance measuring methods is prolific but for general reading the Oxford conference held in 1965 covers many aspects $(60)$. Specific papers dealing with earth movement measured with electronic devices have been published. A programme to study earth movements across faults in Central California has been in progress for many years (61). This has also been described in papers by Scholz and Fitch $(62,63)$.

In each of the above EM methods the current knowledge of the speed of EM waves (the velocity of light) is only marginally superior to field measurements. This is to be expected for the most recent determinations of the velocity of light have been made with similar equipments operating in more ideal situations. Baird has reviewed RF measurements of the speed of light $(64)$. For earth strain measurement the absolute value is not so important provided a common value is used for each comparitive measurement.

\section{Electromagnetic Radiation Methods-Transit Time}

The third method for measuring distance with EM radiation is to time the transit of the wave to a distant station and back, as shown in figure 9. Difficulties of gating clocks with better than nanosecond precision restricts the resolution of this method to many millimetres. At present, therefore, such methods have little application in earth strain measurement except where large deformations occur.

The extreme distance between the moon and the earth and the lack of a velocity modifying atmosphere enables this technique to measure their separation to a part in $10^{8}$. American Astronauts have recently placed banks of retroreflectors on the lunar surface for this purpose. These will assist the determination of relative movements between the two bodies(65).

\section{Very Long Baseline Interferometry (VLBI)}

Another system using interferometry for surface movement monitoring but using radiation from sources already in existence in outerspace, is that of very long baseline interferometry. The angular size of a stellar source can be precisely defined from the combined signals received simultaneously by two or more widely separated telescopes. Radio telescopes located on different continents have been used this way since the early 1960 's to detect and to measure the diameter of newly found sources in space. Measurements have spanned between Britain, Canada, Sweden, Australia and the United States $(66-68)$,

In 1969 it was suggested by workers (69) at the Smithsonian Institute that the situation could be reversed in order to decide the baseline variatigns between telescopes to as small a part in $10^{9}$.

As timings between the two signals are needed to fractional microseconds, methods have been devised using independent recording, figure 10, at each site. The records are then combined to obtain the fringes. VLBI has the potential for investigating earth movements with respect to outer space and between widely separated earth locations.

\section{Application of Techniques}

The inherent feature of the various methods decides to a large extent their appropriate field of application. For mechanical methods environmental temperature and pressure variables alter thelength of the standard (and also perhaps the length being measured). For crustal strain measurements a deep site is essential to avoid surface effects. In deep chambers pressure can be controlled and the temperature gradients corrected for.

The enclosed laser interferometer can be made independant of environmental effects but it would be pointless to use such on the surface for crustal measurements unless it were certain that surface effects were negligible.

The low resolution of EM methods using modulated beams is well matched to the diurnal surface effects although some improvement in instrument precision can be used with effect. Such methods are adequate for measuring fault movements. It will be interesting to see in the future how stable near-surface bench marks are but to date surface measuring equipment has insufficient precision to investigate this.

Although this paper covers strain measurement it is important to remember that the measurement of tilt, that is, change of angle to the horizontal or $\left.{ }_{1}\right)_{\text {rtical may be relevant }}$

\section{Length Standard.}

A brief discussion of length standards is now given as these indicate the upper limits of obtainable accuracy and precision. Special reference is made to decametre standards as these are most appropriate to geophysical strain measurement. 
The international standard of length is the metre which was adopted in 1960 as $1,650,763.73$ wavelengths of radiation in vacuum, corresponding to the transition between the levels $2_{p_{10}}$ and $5_{\mathrm{d}_{5}}$ of an atom of krypton. In vacuum the theoretical limit is better than a part in $10^{8}$.

Due to the relatively short coherence length of the krypton wave only submetre lengths can be established by interferometry. These are then multiplied to produce $1 \mathrm{~m}$ bars. For longer lengths the $1 \mathrm{~m}$ bar is used to produce a $4 \mathrm{~m}$ bar which, in the Societe Genevoise geodetic bases $(70)$ mostly used, is rolled along a track passing under microscopes mounted at $4 \mathrm{~m}$ intervals. The telescopes are used as a transfer mark enabling the bar to be placed end to end with its last location. Once the telescopes are calibrated the unknown length (usually a surveying tape) is calibrated and the base rechecked with the $4 \mathrm{~m}$ bar.

These bases have at the best ${ }^{\circ} \mathrm{C}$ temperature stability and are generally built to about $100 \mathrm{~m}$ in length in vaults beneath the laboratories using them $(7)$.

This cascading procedure realises a little better than a part in $10^{6}$ length precision. To date there has been no real incentive to improve the accuracy. For greater precision the $4 \mathrm{~m}$ bar would need better calibration and closer temperature control would be essential. Ground length changes might also present a problem.

The National Physical Laboratory in Britain are developing a laser interferometer system to measure the absolute length of tapes to greater accuracy and, more importantly, with greater speed. The current method is most laborious and slow.

The absorption stablized lasers, mentioned above, will enable direct lengths to be established with extreme precision. Recommendations were made in 1970 that further work be conducted by National bodies on the methane and the lodine absorption cell laser systems with a view to their eventual adoption as a new, improved, length standard replacing the Krypton method.

\section{Research to Advance Strain-Meter Techniques}

The above review shows that the many methods for measuring earth-strain involve geodetic surveying methods and national standards of 1 ong length.

A current program at the University of New England is concerned with establishing an underground tidal observatory. A mine 1600 feet long, with 1500 feet of cover, has been procured 20 miles from the University at Hillgrove. Power has been installed to the tunnel and telemetry arranged for multiple channels of data to be relayed from the mine to the Geophysics Department's data centre where it can be digitally processed as desired.

In this mine strain meters of solid and flexible mechanical standard design and a laser interferometer are in the process of design and installation. The Bureau of
Mineral Resources (BMR) of Australia is to shortly install horizontal pendulums after Melchior. Requests to site yapour magnetometers and a gravimeter have already been received from northern countries.

In order to investigate the possibilities of thermally controlled standards, temperature stabilizedrods and wires are to be mounted for observation of their own stability and for earth strain recording.

Absolute length measurement of substandards placed on the measuring base will be provided by a variable length evacuated laser interferometer.

A minor but important part of the programme will be the investigation of mounting system stabilities for attaching instruments to the rock. The tunnel length is such that a 0.5 kilometre base could be established for checking geodetic distance measuring devices。

\section{Summary}

Mechanical-standard strain meters are firstly discussed showing that flexible standard methods have great promise for more widespread inexpensive measurements. Inter. ferometric methods, that is, those using the laser, have inbuilt calibration but to date the source reliability is inadequate。 The cost of these instruments excludes them from widespread use. Modulated radiation methods are useful for surface measurement over long distances but they have relatively low resolution and are unable to resolve tides.

The interference length-multiplication method after Vallsalla is still the superior method for absolute length calibration but due to costly equipment and extreme observation times required has not been widely adopted.

The advent of the atomic stabilizedlasers will provide means to establish length measurements to as much as a part in $10^{9}$ precision error provided the atmospheric errors are eliminated.

Very long base radio interferometry has the potential to monitor intercontinental deformations but this work is still in its infancy.

Finally, it is stressed that more effort should be applied to determine the long-period stability of mechanical standards as such instruments are cheap and very reliable.

\section{References}

1. Stacey, Fo D., "Physics of the Earth", John Wiley, Sydney, 1969.

2. Bender, P. L., "Laser measurements of long distances", Proc. IEEE, 1967, 55, 6, $1039-1045$.

3. Prior L. S. Bureau of Mineral Resources, Australia, Private communication 1970.

4. Benioff H., "A Linear Strain Seismograph" Bul1. Seis. Soc. Am. 1935, 25, 4, 283-309.

5. Benioff H., "Fused-quartz Extensometer for Secular, Tidal and Seismic Strains", Bull. Geol. Soc. Am. 1959, 70, 1019-1032.

6. Major M. W., Sutton, G. H., OIiver J., and Metsger R., "On Elastic Strain of the Earth in the period range 5 seconds to 
100 hours", Bull. Seis. Soc. Am., 1964, $54,1,295-346$.

7. Bilham, R. Go, "The Measurement of Earth Strain", Ph。D. dissertation, Cambridge University. Oct。 1970

8. Blayney, J. Lo, and Gilman, R., "A Portable Strain Meter with Continuous Interferometric Calibration"。Bull. Seis. Soc. Am。, 1965, $55,6,955-970$.

9. Major, M. W., "Strain Meters" in ESSA Symp. on Earthquake Prediction February 1966. Washington, D.C. U.S. Printing office 1966.

10. Tomaschek, R., 1957 Encycl. Phys. 48, 815, Springer-Verlag Berlin。

11. Sydenham, P. Ho, "A Length Stabilized 12m measuring base". J. Sci. Instrum, 1969, Ser 2, 2, 523-525.

12. Larsen, N. T., "50 Microdegree temperature Controller", Rev. Sci. Instrum., 1968, 39, $1,1-12$.

13. Harvey, M. E., "Precision TemperatureControlled Water Bath", Rev. Sci. Instrum. $1968,39,1,13-18$.

14. Melchior, P., "The Earth Tides", Pergamon Press, London, 1966.

15. Clark, J. S., "Notes on Geodetic Surveying Tapes", National Physical Laboratory, Internal Report SS 286, 1952.

16. Sydenham, P. H., "A tensioned-wire Strain Seismometer", Jnl. Sci. Instrum. 1969, Ser, 2.2, 1095-1097.

17. Sydenham, P. H., "Tensioned-Wire Length Measuring Instruments", Trans. Inst. Meas. and Control, London, 1969, 2, T194-201.

18. King, G.C.P., Bilham, R. G., Gerard V. B., Davies, Do and Sydenham, P. Ho, "New Strain Meters for Geophysics" Nature, $1969,223,818-819$.

19. Bilham, R. G., Dept. of Geophysics, Cambridge University, England. Private Communications.

20. Gerard V. B., D.S.I.R., New Zealand, paper in progress.

21. Sydenham, P. H., Manual and Automatic Large-Scale Dimensional Metrology", Ph。D. Thesis, 1969, University of Warwick, England.

22. Baird, K. M., "The Role of Interferometry in Long Distance Measurement", Metrologia, $1968,4,3,135-144$.

23. Vali Vo, and Bostrom, R. C., "One Thousand Metre Laser Interferometer", Rev. Sci. Instr. 1968, 39, 9, 1304-1306.

24. Birnbaum G., Frequency Stabilization of Gas Lasers", Proc. IEEE, 1967, 55, 6, 1015-1026.

25. Hall, J. L., "P-1 The Laser Absolute Wavelength Standard Problem", IEEE Trans. $1968, \mathrm{QE}-4,10,638-641$.

26. Hanes G. R., and Baird, K. M., "I2 Controlled $\mathrm{He}-\mathrm{Ne}$ Laser at 633nm: Preliminary Wavelength". Metrologia, 1969, 5, 1, 32-33.

27. Barger R. L. and Hall, J. L., "Pressure Shift and Broadening of Methane Line at 3. 39 studied by laser-saturated Molecular Absorption." Physics Rev. Letters, 1969, $22,1,4-8$.

28. Hanes, G. R. and Dahlstrom, C. E., "Iodine Hyperfine Structure Observed in Saturated Absorption at 633 nm $^{*}$ App. Phys. Letters, 1969, 14, 11, 362-364.

29. Vali, V., and Krogstad, R. S., Measurement of Earth Tides and Continental Drift with Laser Interferometer". Proc. IEEE, $1964,52,857-858$.

30. Vali., V., Krogstad, R. S., and Moss R. W., "Laser Interferometer for Earth Strain Measurements", Rev. Sci. Instrum. 1965,
$36,9,1352-1355$

31. Vali, V., Krogstad, R. So, and Moss R. Wo, "Observation of Earth Tides Using a Laser Interferometer", Jnl. App。Phys.1966, $37,2,580-582$ 。

32. Bostrom R. G., and Vali, V., "Strains Recorded on a High Magnification Interferometric Seismograph" Nature, 1968, 220, $1018-1020$.

33. Cook, A. H., "Gas Lasers and the Measurement of Long Period and Secular Changes in Strain". in S.K. Runcorn "Applications of Modern Physics to Earth and Planetary Interiors", Wiley Interscience, New York 1969.

34. King, G.C.P., Davies, D., "A Laser Seismometer"ib.id.

35. Gerard, V。B., "A Frequency Controlled Laser for Long Path Interferometry". J. Sci。 Instrum. 1969, Ser 2, 2, 749-750。

36. Gerard, V. B., "An Automatic Fringe Follower for a Long Path Laser Vacuum Interferometer". J. Sci. Instrum. 1969, Ser 2, 2, 933-935.

37. Manzoni G。, and Sona, A. "A Long Distance Interferometer for Geodetical Purposes", Symposium on "Electronic Distance Measuring and Atomospheric Refraction", Boulder June 1969 。

38. Boyne, H. So, Hal1, J. L., Barger, R。 L. Bender, P.L., Ward, J., Levine, J., and Faller J。 "Absolute Strain Measurements with a 30 meter vacuum Interferometer" in Laser Applications in The Geosciences" Gauger J. and Hall F. F., Western Periodicals, Calif. 1969.

39. Levine Jo, and Hal1, J. L., "Construction and Operation of a Methane Absorption Stabilised Laser Strain Meter", Internal Report Joint Inst. Lab. Astrophys。, Univ. Colorado, U.S.A.

40. Hall, J. L., Barger, R. L., Bender, P。 L., Boyne, H. S., Faller J. E., and Ward, J. "Precision Long Path Interferometry and the Velocity of Light", Electron Tech. $1969,2,2 / 3,53-66$ 。

41. Shamsi, S. K. and Stacey, F。 D., "Michelson Interferometer as an Earth Strain Senser" Earth Planet Sci. Lett, 1967, 3, 466-468.

42. Vali, V., and Bostrom, R. G., "Some Earth Strain observations with a Thousand Metre Interferometer" Earth Planet Sci. Lett. $1968,4,436-438$.

43. Văisăla Y., "Die Anwendung der Lichtinterferenz zu Längen Messupgen Auf Grösseren Distanzen", Veroff.d.Finn.Geod. Inst. No. 2。 Helsinki 1923.

44. Honkasalo T. MInternational Standard Base Lines" in Geodoettinen Laitos (Finnish Geodetic Institute) No. 65 Helsinki 1969 , $57-67$.

45. Kukkamäki, T. J., "Ohio Standard Baseline" Ann. Acad. Scient. Fennicae, 1969, A, III, $102,1-60$.

46. Skogorev, V. P. "Review of Experience in Distance Measurement by Light Interference Method", Geod。 and Aerophot, 1968, No。3, 208-211.

47. Negri H。, "Vâisälä light Interference Comparator - a Valuable Contribution to the Geodesy of Today" Ann. Acad. Scient. Fennicae, 1961, Ser. A, III, 61, 169-184.

48. Honkosalo $\mathrm{T}$. "Measuring of the 864m-1ong Nummela Standard Base Line with the Våsalä Light Interference Comparator and some Investigations into Invar Wires", Suomen Geodeettisen Laitoksen Julkaisuja Ver $8 f f e n t 1 i c h u n g e n$ des Finnischen 
10

Geodeatischen Institutes, 1950, No. 3?.

49. Vailsale Y. and Oterma L. "System of quartz Metres and the Absolute Length of its Gauges", Metrologia, 1967, 3, 37-41.

50. Clark D. "Plane and Geodetic Surveying Vol II, Higher Surveying (Constable, 1963).

51. Bradsel1, R. H. "Measuring Distance with the Mekometer". New Scientist, 1962, 297, 206-207.

52. Froome $K$. D. and Bradsell R. H. A New Method for the Measurement of Distances up to 5000 feet by Means of a Modulated Light Beam" J.Sci。Instrum., 1966, 43, 129-133。

53. Owens J. C. and Earnshaw K. B., "Long Distance Optical Strain Meters for Fault Zone Instrumentation" PROC ESSA Symp" Earthquake Prediction 1965, Rockvilie, U.S.A.

54. Thompson M, C. and Wood L. E. The use of Atmospheric Dispersion for the Refractive Index Correction of Optical Distance Measurements" in "Electromagnetic Distance Measurement" Symp.1965, Oxford (Hilger and Watts).

55. Thompson Mo C. and Janes Ho B., Correction of Atmospheric Errors in Electronic Measurements of Earth Crustal Movements Bull. Seis. Soc. Am, 1967, 57, 4, 641-655.

56. Owens $J_{0} C_{\text {. }}$ and Earnshaw K. B. Development of a Microwave Modulated, Dual optical Wavelength Geodetic Distance - Measuring Instrument, ESSA Tech. Rep., 1968, No. ERL 117-WP1 8, Colorado.

57. Owens J. C. "A review of Atmospheric Dispersion Measurements in Geodesy and Meteorology Conf. Refraction effects in Geodesy and Electronic Distance Measurement", 1968, Univ. New South Wales, Australia.

58. McQuistan G.W. Electronoquetic Distance Measuring with Two Wavelengths - Atmospheric limitations"ib id.

59. Froome K. D. Assessment of Mekometer III from Geophysical Measurements in Iceland". Symp. Electronic Distance Measuring and Atmosphere Refraction" Boulder, Colarado, U.S.A., 1969, also private communications, R. G. Mason, Geophysics Dept. Imp. College.

60. "Electromagnetic Distance Measurement (EDM) Hilger and Watts, 1967.

61. Whitten C. A. Weasurement of Small Movements in the earth's crust" Ann. Acad。 Scient. Fennicae, 1961, A, III, 61, 315 320 .

62. Scholz C. H, and Fitch T. J., Strain Accumulation along the San Andreas Fault InI. Geoph. Res. 1969, 74, 27, 6649-6666

63. Scholz C. H. and Fitch T. J. "Strain and Creep in Central California", Jnl. Geoph. Res. 1970, 75, 23, 4447-4453.

64. Baird R. C., RF Measurements of the speed of Light, Proc. IEEE, 1967, 55, 6, 1032 . 1039 .

65. Alley, Co O., Bender, P. L, Currie, D. G., Dicke, R. H. and Faller J. E. Some Implications for Physics and Geophysics of Laser Range Measurements from Earth to a Lunar Retro-Reflector" in "Applications of Modern Physics to Earth and Planetary Interiors 1969.

66. Cohen M. H. Jauncey D. L. Kellermann K.I. and Clark B. G." "Radio Interferometry at one-thousandth second of arc", Science $1968,162,88.94$.

67. Gubbay J., Legg, A. J., Robertson, D. S., Moffet A. T. and Seidel B. Trans-pacific
Interferometer Measurements at $2300 \mathrm{MHz}^{\text {s }}$ Nature, 1969, 222, 5195, 730-733.

68. Locke Jo Lo Very Long Base1ine Interferometry Science Jn1. 1969, 41-46.

69. V.L.B.I. New Geodetic Technique" SAO News 1969.

70. Martin P. "Metrology" Int。 Repo Societe Genervise D'instruments de Physique" 1967, No. 1314, Geneva. 


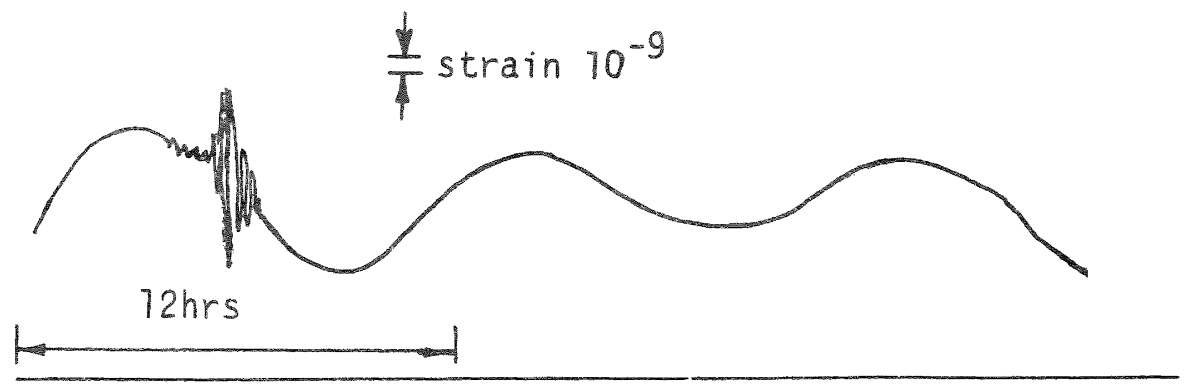

Fig. 1. Representative tidal curve with magnitude 6 earthquake.

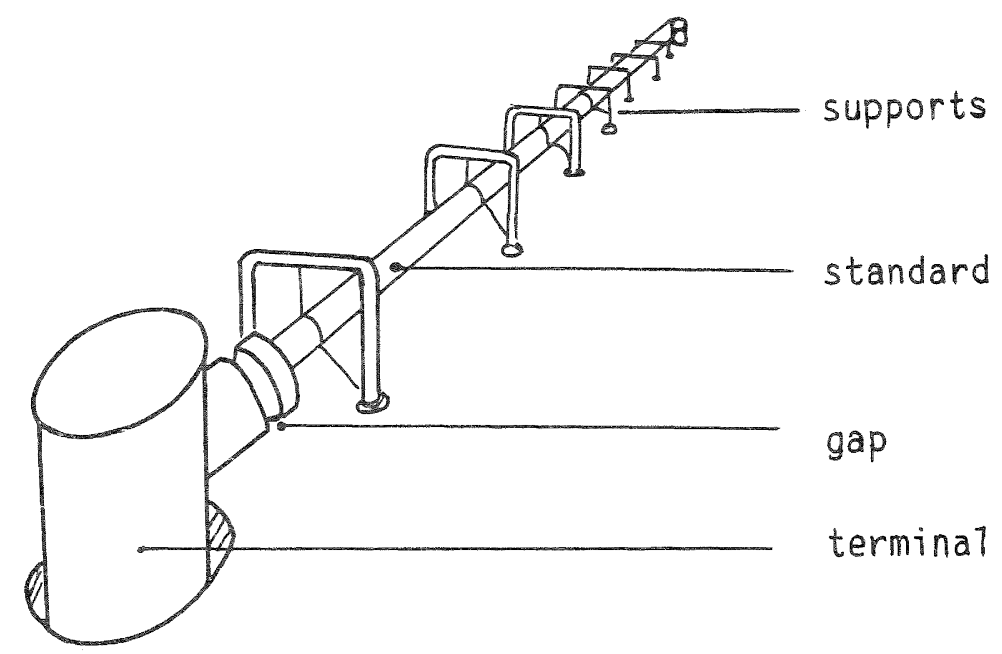

Fig. 2. The original steel standard Benioff gauge ${ }^{10}$.

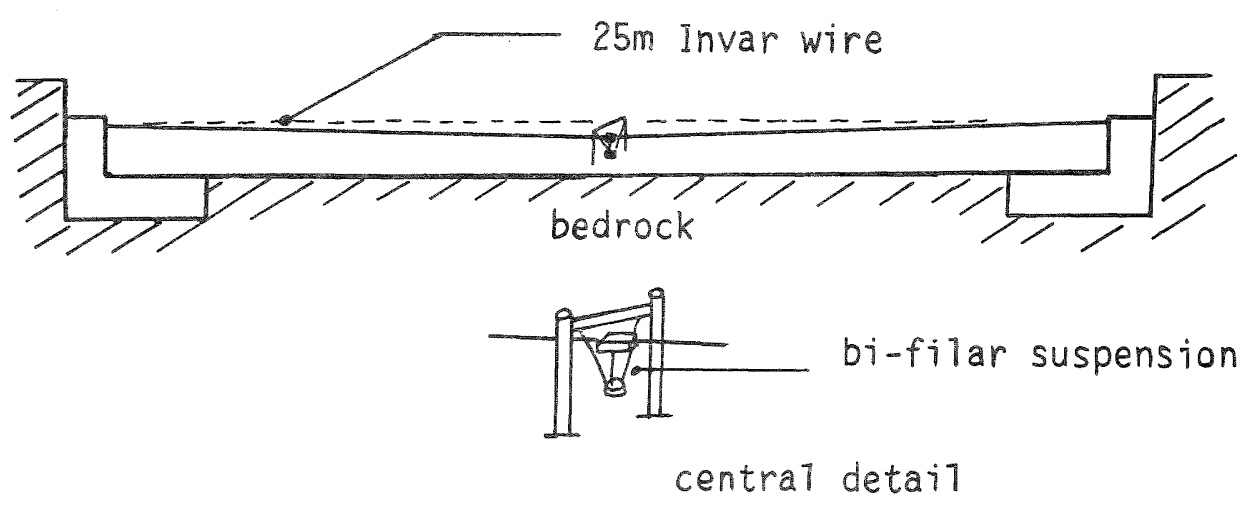

Fig. 3. Schematic of the Sassa extensometer ${ }^{10}$. 


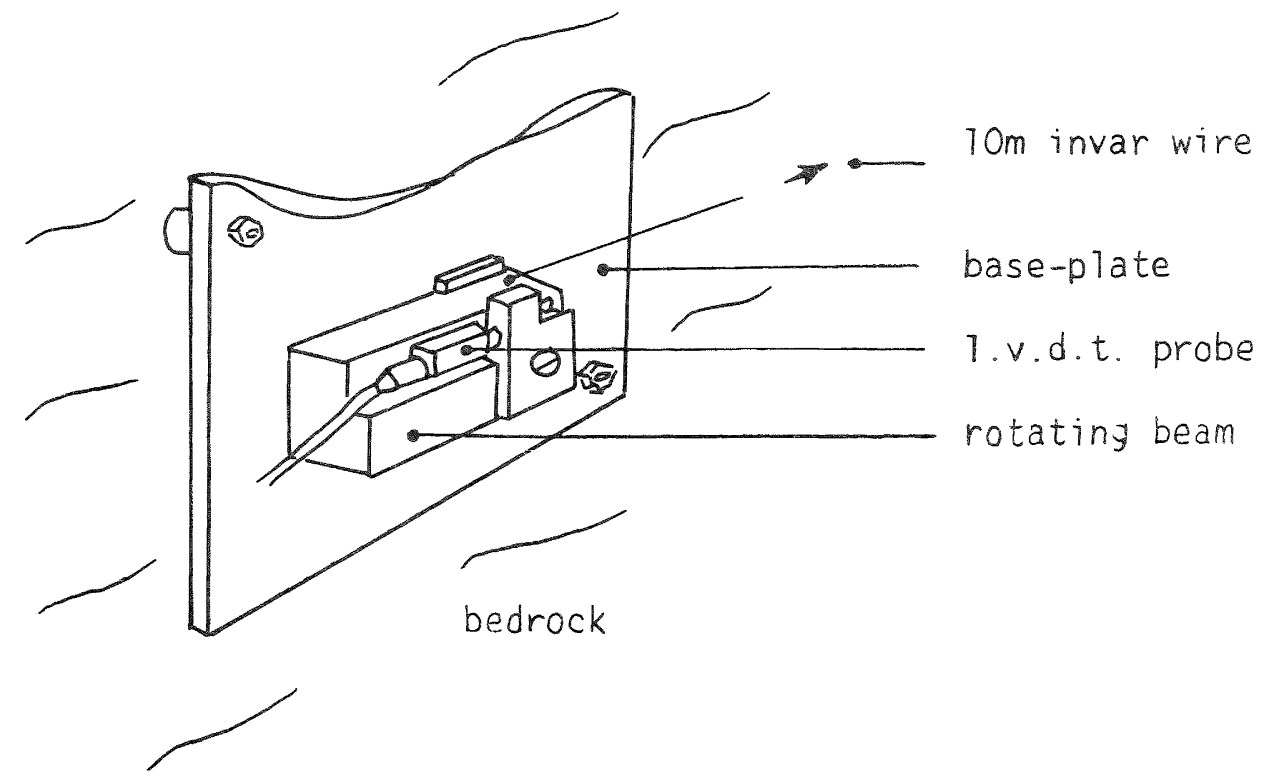

Fig. 4. The original Sydenham strain seismometer"

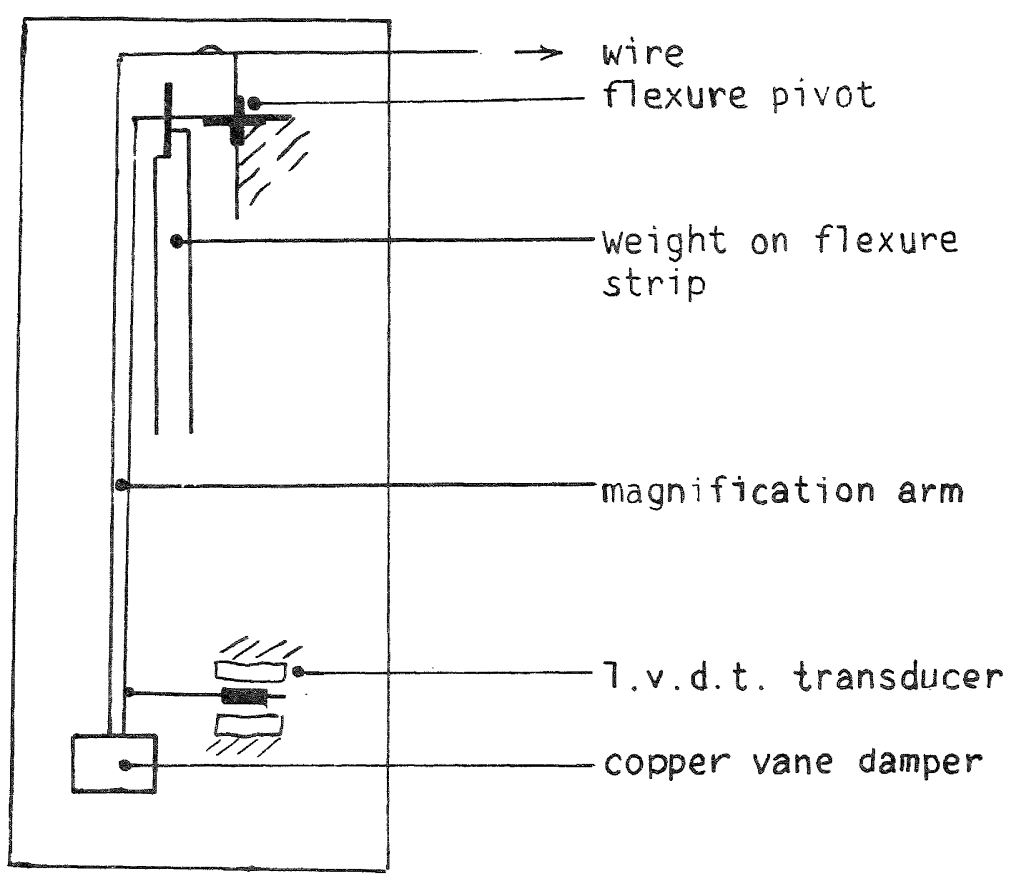

Fig. 5. The Theometer of Cambridge University 


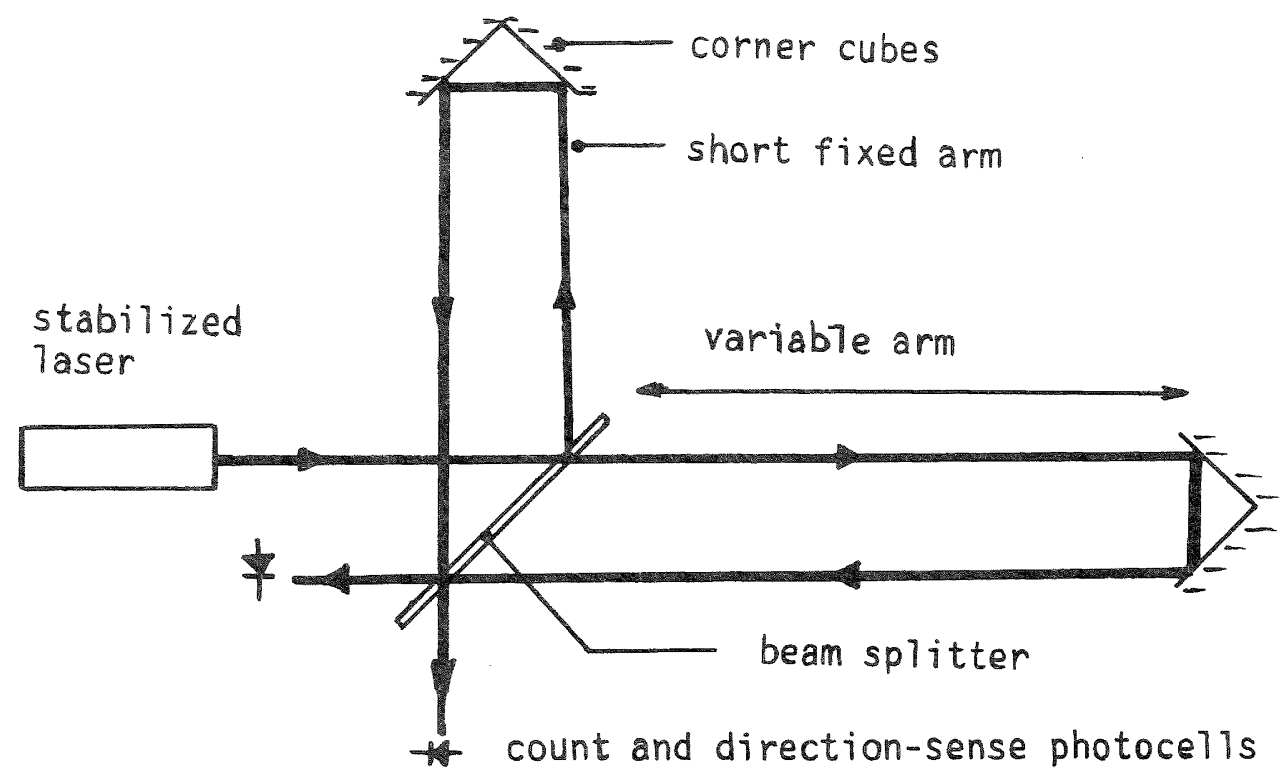

Fig. 6. Optical layout of the Michelson laser interferometer strain meter.

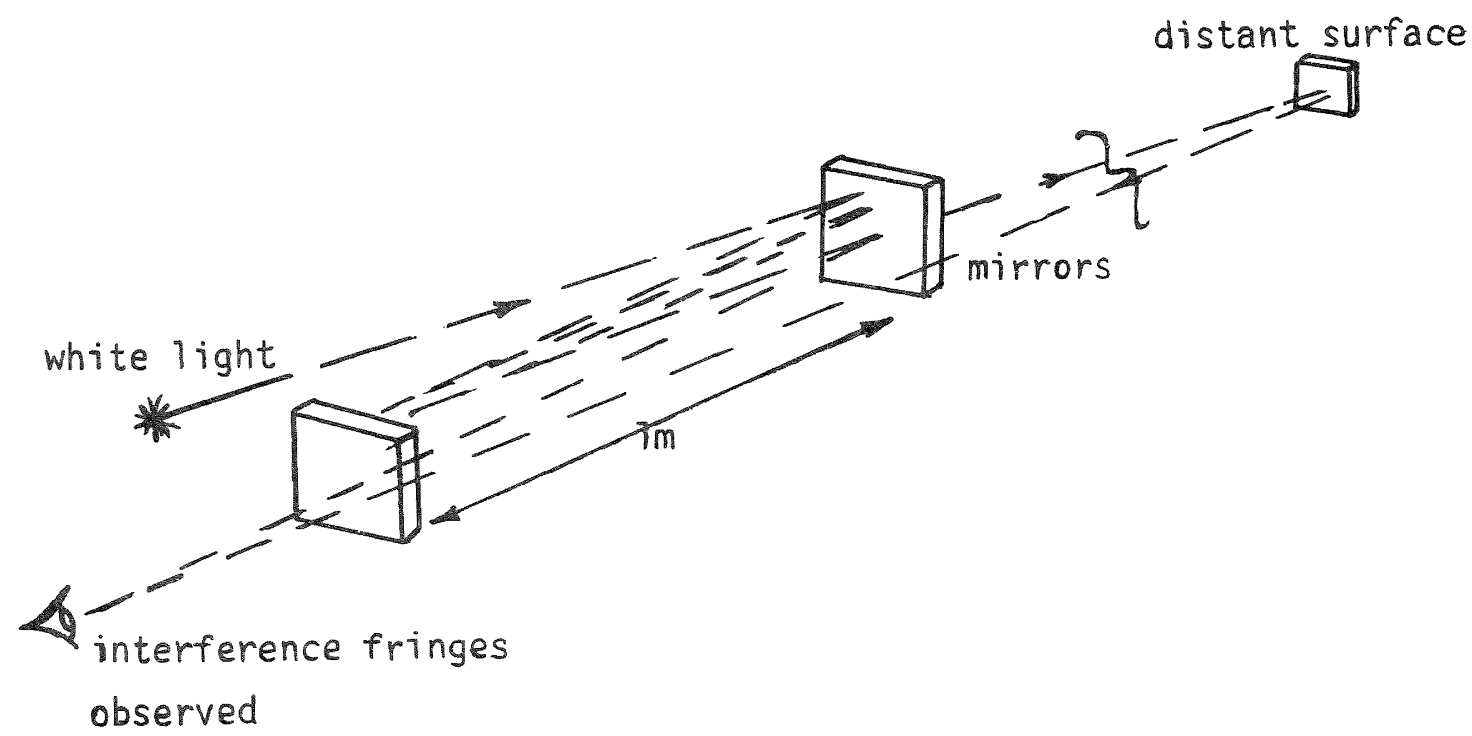

Fig. 7. Schematic of the Vaisala interference multiplication method. 


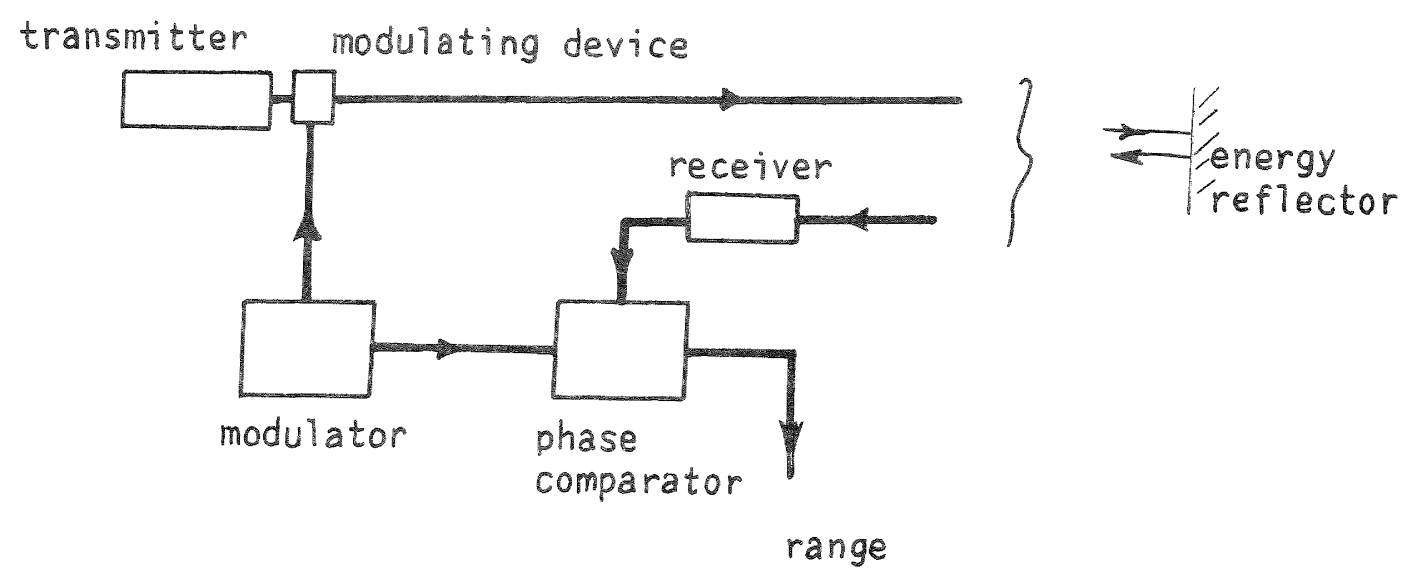

Fig. 8. Electromagnetic distance measurement using a continuously modulated beam.

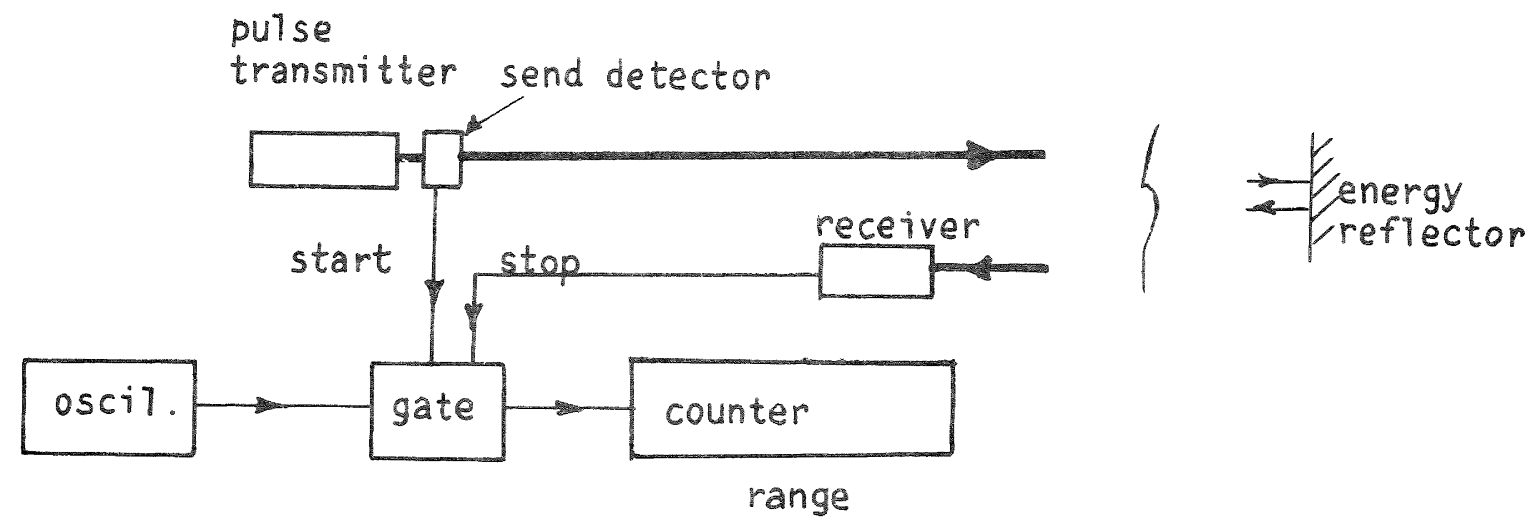

Fig. 9. Transit time pulsed distance measurement.

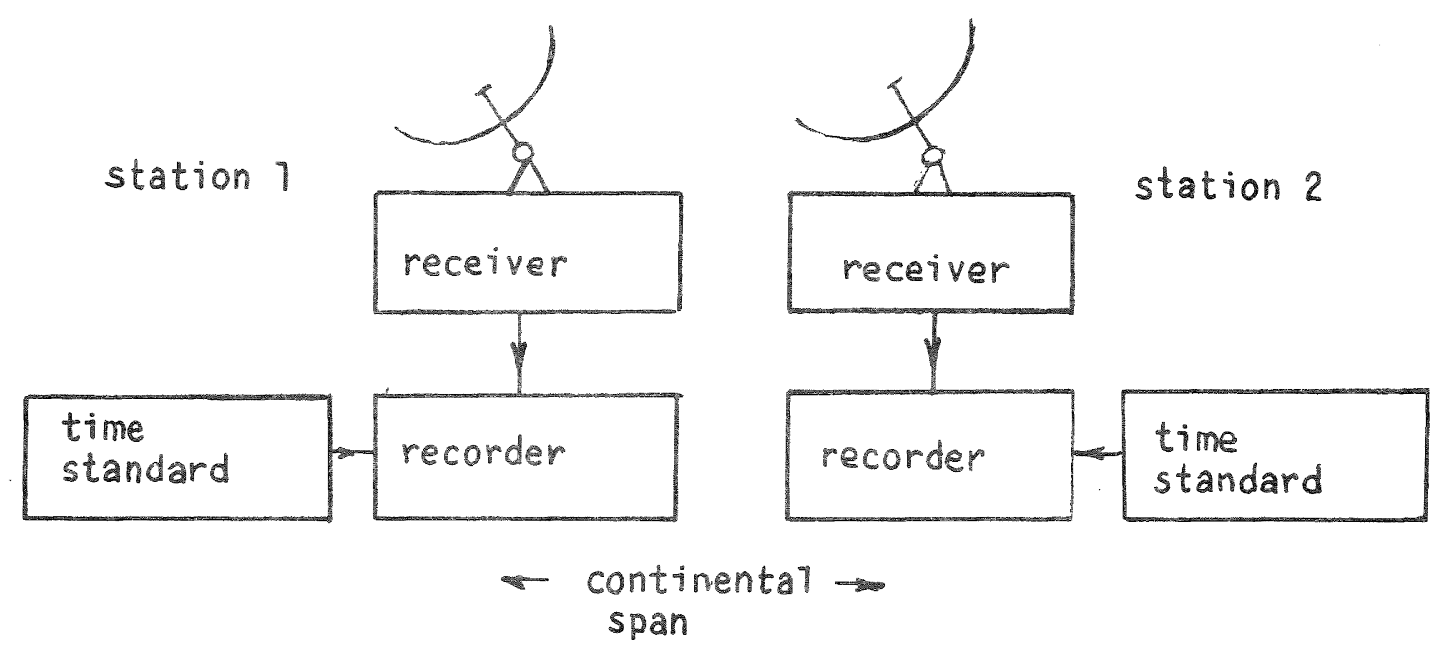

Fig. 10. Very long baseline interterometry using independant recording. 